\title{
Prevalencia de fracturas faciales atendidas en el servicio de cirugía maxilofacial del Hospital Carlos Andrade Marín HCAM entre los años 2013 y 2018 Prevalence of Facial Fractures Served in the Maxillofacial Surgery Service of the Carlos Andrade Marín HCAM Hospital Between 2013 and 2018
}

\author{
Juan Diego Salazar ${ }^{1}$ \\ Fernando J. Sandoval P. ${ }^{2}$ \\ Fernando Sandoval V. ${ }^{3}$
}

\begin{abstract}
Universidad San Francisco de Quito, Colegio de Ciencias de la Salud, Escuela de Odontología, Clínica Odontológica, Campus Cumbayá, Oficina C0 106, casilla postal 17-1200-841. Quito-Ecuador. Correo electrónico: juandieguito87@aol.com 2Universidad San Francisco de Quito, Colegio de Ciencias de la Salud, Escuela de Odontología, Clínica Odontológica, Campus Cumbayá, Oficina C0 106, casilla postal 17-1200-841. Quito-Ecuador. Correo electrónico: fjose18@hotmail.com ${ }^{3}$ Universidad San Francisco de Quito, Colegio de Ciencias de la Salud, Escuela de Odontología, Clínica Odontológica, Campus Cumbayá, Oficina C0 106, casilla postal 17-1200-841. Quito-Ecuador. Correo electrónico: fsandoval@usfa.edu.ec
\end{abstract}

Editado por / Edited by: Johanna Monar

Recibido / Received: 06-08-2019

Aceptado / Accepted: 24-09-2019 


\section{Resumen}

El trauma se define como una lesión física generada por la aplicación de una fuerza externa, en contra del cuerpo humano; al trauma facial se lo considera una de las patologías más frecuentemente tratadas en los departamentos de cirugía oral y maxilofacial, representando la mayor parte de los pacientes que han sufrido algún tipo de traumatismo, convirtiendo a este en un problema trascendental para salud pública. Las lesiones faciales se las considera complicadas por su cercanía a estructuras anatómicas importantes, los cuales se pueden ver afectados por el traumatismo en primer lugar, dejando secuelas que en ocasiones resultan imposibles de solucionar. El objetivo de este estudio fue establecer la frecuencia de las fracturas del macizo facial así como su etiología y relación con la edad, genero, frecuencia, localización anatómica en los pacientes tratados en el servicio de maxilofacial del hospital Carlos Andrade Marín, para así poder aportar información estadística real y actualizada en base a la magnitud, naturaleza, causas y manejo de la fracturas faciales en el ámbito regional y así proveer una mejor percepción del trauma maxilofacial y su origen y así ayudar a la toma de medidas preventivas y terapéuticas. Un total de 432 pacientes sufrieron lesiones maxilofaciales de los cuales la mayoría se produjo entre la tercera y cuarta década de la vida con una relación de 9:1 entre hombres y mujeres, la causa más común fueron las agresiones físicas $(28,50 \%$ ) seguido por los accidentes en motocicleta $(22,20 \%)$. La factura más frecuente fue la mandibular con un $32,4 \%$ seguido por fracturas del complejo orbito cigomático maxilar con un $28,5 \%$ y las fracturas del piso de orbita $(14,1 \%)$ (Tabla 8$)$.

Palabras Clave: Trauma facial, prevalencia, fractura maxilar, fractura mandibular

\section{Abstract}

The trauma is defined as a physical injury generated by the application of an external force, against the human body, inside this is the facial trauma is considered one of the most frequently treated pathologies in the oral and maxillofacial surgery departments, representing these the most of the patients who have suffered some type of traumatism making this a transcendental problem for public health. Facial injuries are traumatisms that rarely become fatal, but they are considered complicated due to their proximity to important anatomical structures, which can be affected by trauma in the first place, leaving sequels that are sometimes impossible to solve. The objective of this study is to establish the frequency of facial mass fractures as well as their etiology and relationship with age, gender, frequency, anatomical location in patients treated in the maxillofacial service of the Carlos Andrade Marín hospital, in order to provide real and updated statistical information based on the magnitude, nature, causes and management of facial fractures in the regional area and thus provide a better perception of maxillofacial trauma and its origin and thus help preventive and therapeutic measures. A total of 432 patients suffered maxillofacial injuries of which the majority occurred between the third and fourth decade of life with a ratio of 9: 1 between men and women, the most common cause being physical aggression (28.50\%) followed by motorcycle accidents $(22.20 \%$ ). 
The most frequent bill was the mandible with $32.4 \%$ followed by fractures of the maxillary zygomatic orbital complex with $28.5 \%$ and fractures of the orbital floor $(14.1 \%)$.

Keywords: Facial trauma, prevalence, maxillary fracture, mandibular fracture.

\section{Introducción}

El trauma se define como una lesión física generada por la aplicación de una fuerza externa, en contra del cuerpo humano' ${ }^{1}$, a este se lo considera una de las patologías más frecuentemente tratadas en los departamentos de cirugía oral y maxilofacial ${ }^{2}$, representando estos la mayor parte de los pacientes que han sufrido algún tipo de traumatismo convirtiendo a este en un problema trascendental para salud pública ${ }^{3}$, debido a la elevada probabilidad de causar una grave morbilidad como desfiguramiento, limitación de funciones como el habla, masticación, e incluso la habilidad de interactuar con otras personas lo que puede derivar en alteraciones psicológicas y sociales importantes ${ }^{4}$, lo cual además de producir un problema para la salud pública puede llevas a problemas económicos ${ }^{5,6}$.

Las lesiones faciales son traumatismos que en raras ocasiones llegan a ser fatales, pero se los considera complicadas por su cercanía a estructuras anatómicas importantes ${ }^{7}$, como las fosas nasales, los senos paranasales, la gran cantidad de estructuras vasculares y nerviosas, las cavidades orbitarias, el cerebro, los cuales se pueden ver afectados por el traumatismos en primer lugar o en un segundo plano por alguna infección derivada es este ${ }^{8}$, dejando secuelas que en ocasiones resultan imposibles de solucionar ${ }^{9}$

La incidencia y etiología de las fracturas faciales varia ampliamente dependiendo de la región geográfica y es afectada por múltiples factores ${ }^{3}$, incluyendo la densidad poblacional, estilo de vida, cultura, estado socioeconómico, el tiempo y las influencias temporales y climáticas ${ }^{4}$, además de las características de la población estudiada como el género, edad el consumo de alcohol, drogas, violencia domestica así como condiciones clínicas como la osteoporosis, son factores que se deben tomar en cuenta al estudiar la epidemiologia del trauma ${ }^{5,10}$.

El diagnostico en este tipo de fracturas debe ser oportuno y preciso, debido a que las opciones de tratamiento se basan en factores como, la edad del paciente, el tipo de fractura, las posibles complicaciones que se puedan presentar ${ }^{11,12}$. De la misma manera, el tratamiento de las fracturas puede variar dependiendo del tipo, este puede ir desde una reducción cerrada con fijación intermaxilar, hasta reducción abierta, la cual permite una adecuada fijación de los fragmentos óseos ${ }^{13,9}$. Un análisis epidemiológico de traumatismo maxilofacial es extremadamente importante para identificar la cinemática del trauma y ayudar a un sistema más eficiente de prevención de las lesiones faciales y el establecimiento de un plan de tratamiento adecuado ${ }^{3,4,7}$. 
Actualmente, existe un déficit de información estadística real y actualizada en base a la magnitud, naturaleza, causas y manejo de la fracturas faciales en el ámbito regional lo que dificulta la obtención de una percepción del trauma maxilofacial y su origen, lo que pudiese ayudar a la toma de medidas preventivas y terapéuticas. Por lo tanto, el objetivo de este estudio fue establecer la frecuencia de las fracturas del macizo facial así como su etiología y relación con la edad, genero, frecuencia, localización anatómica en los pacientes tratados en el servicio de cirugía maxilofacial del hospital Carlos Andrade Marin ${ }^{14}$.

\section{Materiales y métodos}

Se realizó un estudio retrospectivo que incluyo pacientes que hayan sufrido traumatismos en la región facial y fueron tratados quirúrgicamente para la reducción y fijación de fracturas dentro del servicio de cirugía maxilofacial del hospital Carlos Andrade Marín.

La muestra se constituyó por los pacientes que sufrieron fracturas faciales y que fueron tratados en el servicio de cirugía maxilofacial del hospital Carlos Andrade Marín en el periodo comprendido entre enero 2013 a enero 2018 y fueron manejados quirúrgicamente. Se tomaron en cuenta la edad, el género, la causa del traumatismo accidentes de automovilísticos, accidentes en motocicleta, caídas, agresiones físicas, traumatismos deportivos, lesiones por armas de fuero, atropellamiento; la región de la lesión, el tratamiento realizado. El análisis no incluyo el tiempo de hospitalización, tiempo de intervención, las complicaciones posoperatorias.

Los criterios de exclusión fueron todos los pacientes que no contaron con historias clínicas completas o que no recibieron tratamiento en el hospital.

Los datos obtenidos se registraron a través de una ficha de observación, con un diseño adecuado que respete los requisitos necesarios para la obtención precisa de información necesaria y útil para esta investigación.

Se realizó una base de datos para recolectar la información en donde se agruparon las variables a estudiar. Posterior a esto se procedió al análisis estadístico y a la graficación de las variables investigadas y finalmente se realizó la interpretación de los resultados y gráficos. Este análisis se realizó mediante el software estadístico IBM SPSS 21.0 para Windows; SPSS, Inc, Chicago, IL.

\section{Resultados}

Un total de 432 pacientes sufrieron lesiones maxilofaciales de los cuales la mayoría se produjo por accidentes de tránsito seguido por actividades deportivas, violencia física, accidentes laborales.

La edad de los pacientes al momento del traumatismo vario desde el año de edad hasta los 86 años, con una mediana de 30 y una media de 34 años, 7,9 \% de los 
pacientes tuvieron menos de 15 años de edad, el $40 \%$ de los individuos estuvieron entre los 21 y los 30 años de edad mientras que el 20,6\% entre los 31 y 40 años, por lo que se puede deducir que la mayoría de los pacientes se presentan entre la tercera y cuarta década de la vida (Tabla 1).

\begin{tabular}{c|c|c} 
& \multicolumn{3}{|c}{ Edad Agrupada } \\
\hline $1-10$ & Frecuencia & Porcentaje \\
\hline $11-20$ & 22 & 5,1 \\
\hline $21-30$ & 30 & 6,9 \\
\hline $31-40$ & 173 & 40,0 \\
\hline $41-50$ & 89 & 20,6 \\
\hline $51-60$ & 52 & 12,0 \\
\hline $61-70$ & 29 & 6,7 \\
\hline $71-80$ & 19 & 4,4 \\
\hline $81-90$ & 16 & 3,7 \\
\hline Total & 2 & 0,5 \\
\hline
\end{tabular}

Tabla 1. Edad Agrupada

La distribución por edad de los pacientes mostró tasas decrecientes de accidentes en cada década de la vida a partir de los 40 años de edad mientas que antes de los 40 años de edad presentaron una incidencia incremental, esto se lo puede observar en los datos obtenidos en los cuales el $52 \%$ de las lesiones ocurrieron en las tres primeras décadas de vida.

Además, los traumatismos por caídas de propia altura predominaron en la primera década de la vida, mientras que los accidentes en motocicleta y las agresiones físicas predominan entre los 20 y 30 años. Estas causas disminuyeron conforme aumentaba la edad de los pacientes y eran reemplazadas por caídas de propia altura la cual fue la causa predominante a partir de los 50 años (Tabla 2). 


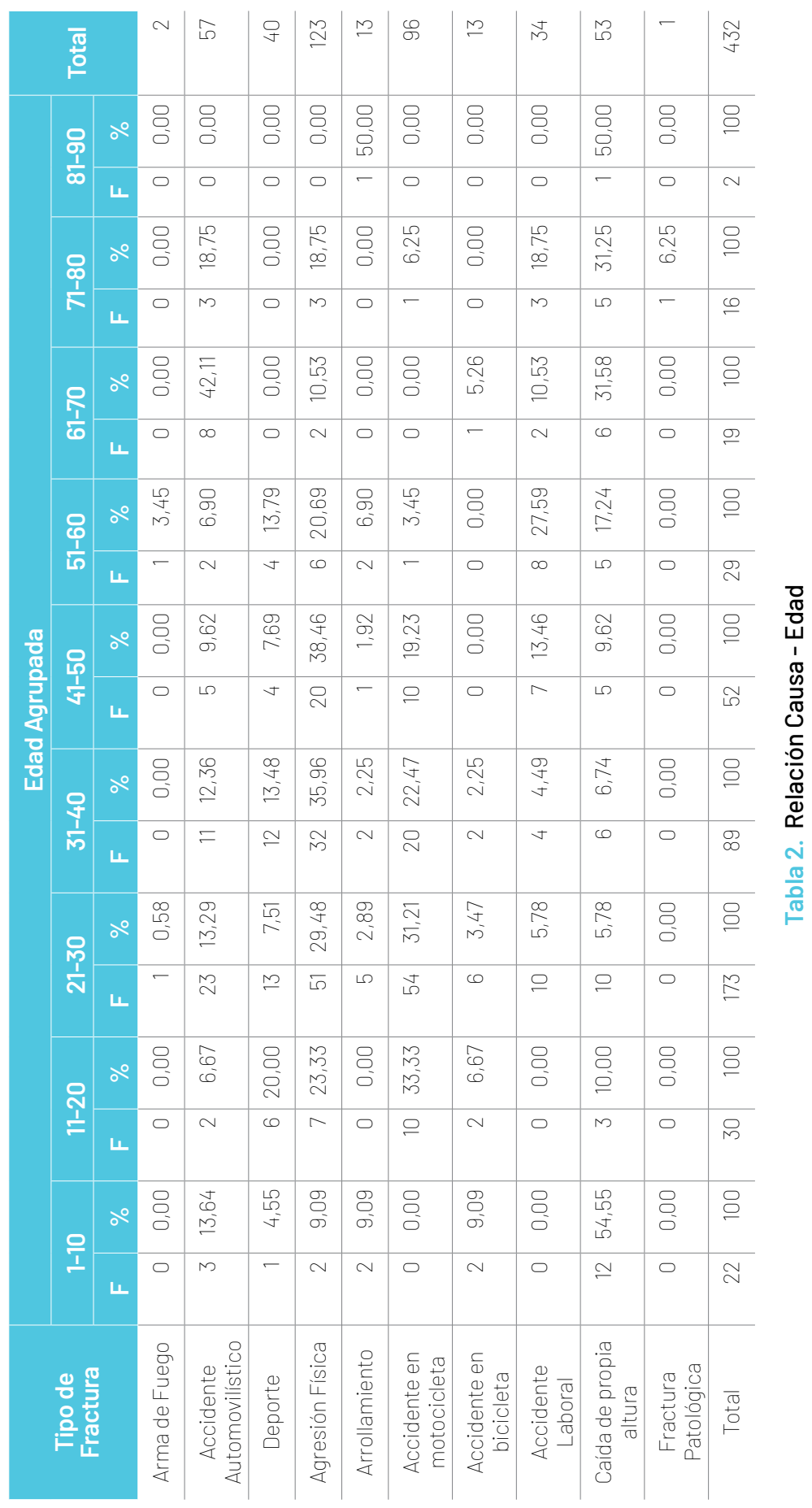


La prevalencia en cuanto a genero fue de 9:1 para los hombres (386) comparado con las mujeres (46) (Tabla 3). Los hombres presentaron una mayor prevalencia que las mujeres en todas las edades, con un riesgo casi 9 veces mayor en la segunda y tercera década de la vida. Los hombres presentaron como la causa de mayor prevalencia las agresiones físicas $(29,79 \%)$ y los accidentes automovilísti$\cos (22,54 \%)$, por otro lado, en las mujeres la mayor causa de fracturas faciales fueron los accidentes de tránsito $(26,09 \%)$ seguido de las caídas de propia altura $(21,24 \%)$ (Tabla 4$)$

\begin{tabular}{c|c|c}
\multicolumn{3}{|c}{ Género } \\
\hline & Frecuencia & Porcentajes \\
\hline Masculino & 386 & 89,4 \\
\hline Femenino & 46 & 10,6 \\
\hline Total & 432 & 100 \\
\hline
\end{tabular}

Tabla 3. Género

\begin{tabular}{l|c|c|c|c|c|}
\multirow{2}{*}{\multicolumn{1}{c}{ Causa }} & \multicolumn{5}{c}{ Genero } \\
\cline { 2 - 6 } & \multicolumn{2}{c|}{ Masculino } & \multicolumn{2}{c}{ Femenino } & Total \\
\cline { 2 - 6 } & Frecuencia & $\%$ & Frecuencia & $\%$ & 2 \\
\hline Arma de fuego & 1 & 0,26 & 1 & 2,17 & 57 \\
\hline Accidente automovilístico & 45 & 11,66 & 12 & 26,09 & 40 \\
\hline Deporte & 38 & 9,84 & 2 & 17,39 & 123 \\
\hline Agresión Física & 115 & 29,79 & 8 & 0,00 & 13 \\
\hline Arrollamiento & 13 & 3,37 & 0 & 19,57 & 96 \\
\hline Accidente en motocicleta & 87 & 22,54 & 9 & 2,17 & 13 \\
\hline Accidente en bicicleta & 12 & 3,11 & 1 & 6,52 & 34 \\
\hline Accidente laboral & 31 & 8,03 & 3 & 21,74 & 53 \\
\hline Caída de propia altura & 43 & 11,14 & 10 & 0,00 & 1 \\
\hline Fractura patológica & 1 & 0,26 & 0 & 100 & 432 \\
\hline Total & 386 & 100 & 46 & \\
\hline
\end{tabular}

Tabla 4. Relación Causa - Género 
De todos los pacientes tomados en cuenta en este estudio se pudo constatar que la mayoría de los traumatismos manejados en el servicio de cirugía oral y maxilofacial del hospital Carlos Andrade Marín la mayoría se produjeron por agresiones físicas $(28,50 \%)$ lo que contrasta con los resultados obtenidos por estudios similares en los cuales los accidentes en motocicleta fueron la causa principal, el cual en nuestro estudio este es la segunda causa (22,20\%)(Tabla 5). En cuanto a las agresiones físicas el tipo de fractura que más se produjo fueron las fracturas del complejo orbito cigomático maxilar $(30,08 \%$ ) y las fracturas del piso de orbita $(22,76 \%$ ), dejando a las fracturas de la mandíbula en tercer lugar (19,51\%)(Tabla 6), de la misma manera las fracturas del complejo orbito cigomático maxilar $(27,08 \%)$ fueron la primera región en sufrir lesiones en los accidentes en motocicleta pero en este caso en segundo lugar se encontraban las fracturas mandibulares $(22,92 \%)$.

\begin{tabular}{l|c|c}
\multicolumn{3}{c}{ Causa } \\
\hline Arma de Fuego & Frecuencia & Porcentaje \\
\hline Accidente Automovilistico & 2 & 0,50 \\
\hline Deporte & 57 & 13,20 \\
\hline Agresión Física & 40 & 9,30 \\
\hline Atropellamiento & 123 & 28,50 \\
\hline Accidente en motocicleta & 13 & 3,00 \\
\hline Accidente en Bicicleta & 96 & 22,20 \\
\hline Accidente Laboral & 13 & 3,00 \\
\hline Caida de Propia Altura & 34 & 7,90 \\
\hline Fractura Patológica & 53 & 12,30 \\
\hline Total & 1 & 0,20 \\
\hline
\end{tabular}

Tabla 5. Causa 


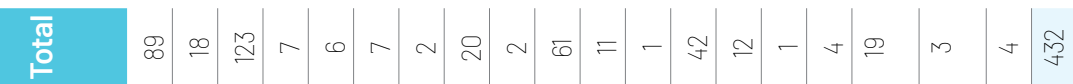

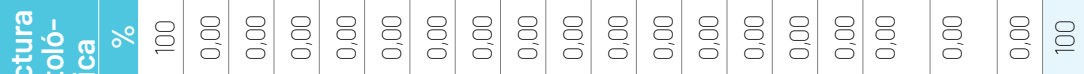

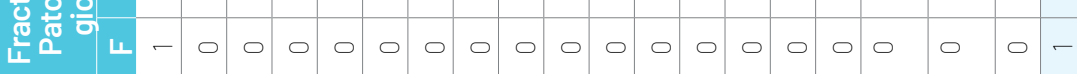

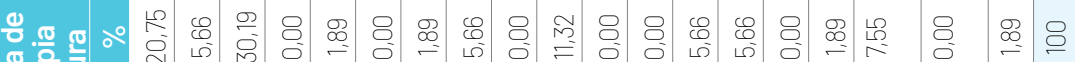
응 응

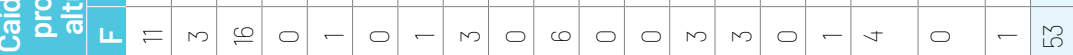

长 。

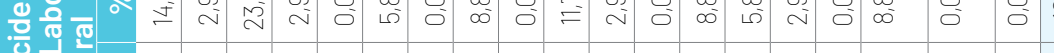

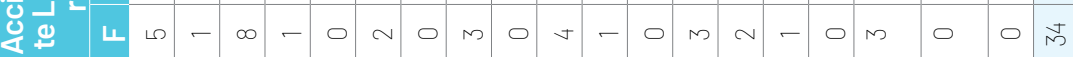

立

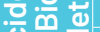

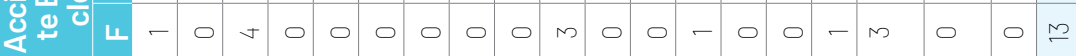

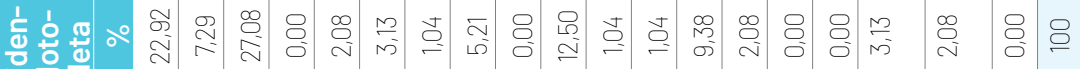
(8)

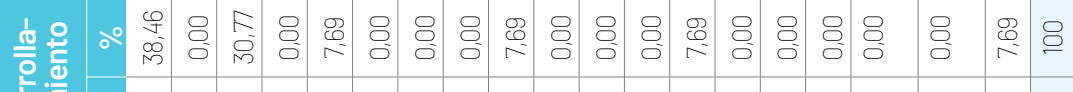
选至 L

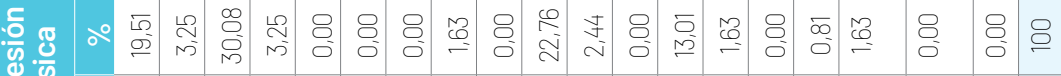

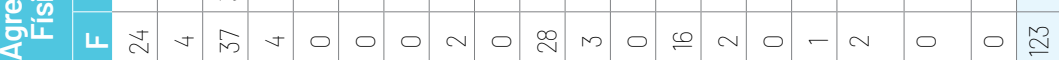

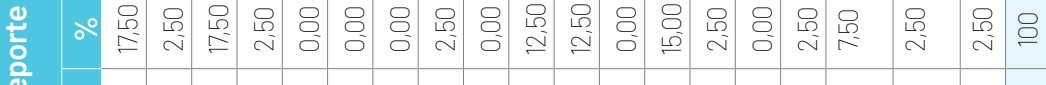

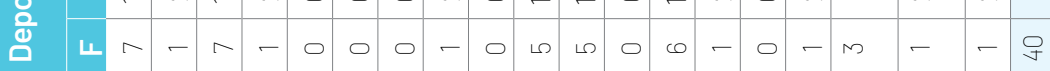

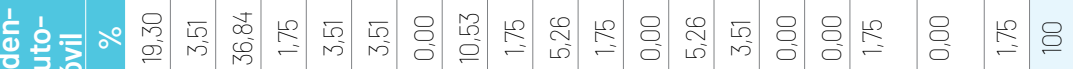
。ํㅠㄹ

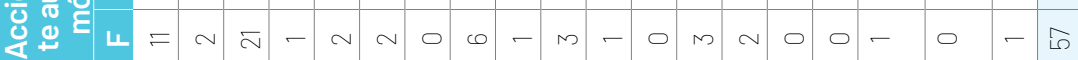

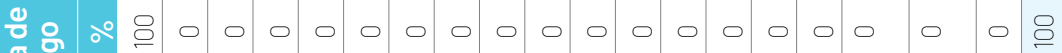

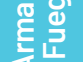
每考 L n 융 똘 을 함 
Un total de 432 pacientes, de los cuales el $3 \%$ presentaron fracturas del tercio superior, $64,6 \%$ fracturas del tercio medio y el $32,4 \%$ presentaron fracturas del tercio inferior facial (Tabla 7). La factura más frecuente fue la mandibular con un $32,4 \%$ seguido por fracturas del complejo orbito cigomático maxilar con un $28,5 \%$ y las fracturas del piso de orbita $(14,1 \%)$ (Tabla 8 ). Las fracturas de mandibulares se produjeron con mayor frecuencia en la segunda década de la vida y la causa más común fue la agresión física y los accidenten en motocicleta, mientras que las fracturas del complejo orbito cigomático maxilar fueron al igual que las fracturas mandibulares, las agresiones físicas seguido de los accidentes automovilísticos.

\begin{tabular}{c|c|c}
\multicolumn{3}{|c|}{ Tercio Facial } \\
\hline Superior & Frecuencia & Porcentaje \\
\hline Medio & 13 & 3,00 \\
\hline Inferior & 279 & 64,60 \\
\hline Total & 430 & 32,40 \\
\hline
\end{tabular}

Tabla 7. Tercio Facial

\begin{tabular}{l|c|c}
\multicolumn{2}{c}{ Tipo de Fractura } \\
\hline Mandibula & Frecuencia & Porcentaje \\
\hline Maxilar & 89 & 20,60 \\
\hline COCM & 18 & 4,20 \\
\hline Techo de orbita & 123 & 28,50 \\
\hline Le Fort I & 7 & 1,60 \\
\hline Le Fort II & 6 & 1,40 \\
\hline Le Fort III & 7 & 1,60 \\
\hline Cóndilo & 2 & 0,50 \\
\hline Panfacial & 20 & 4,60 \\
\hline Piso de Orbita & 2 & 0,50 \\
\hline Angulo Mandibular & 11 & 14,10 \\
\hline NoE & 1 & 2,50 \\
\hline Arco Cigomático & 42 & 0,25 \\
\hline Parasinfisiaria & 12 & 9,70 \\
\hline Rama Mandibular & 1 & 2,80 \\
\hline Frontal & 432 & 0,25 \\
\hline Dentoalveolar Maxilar & 4,90 \\
\hline Dentoalveolar Mandibular & 190 \\
\hline Cuerpo Mandibular & 11 & 0,90 \\
\hline
\end{tabular}

Tabla 8. Tipo de Fractura 


\section{Discusión}

La primera causa de muerte en los primeros 40 años de vida son los traumatismos $^{15}$. Además las lesiones traumáticas han sido identificadas como una de las mayores causas de baja productividad, causado más perdidas de trabajo al año de que las enfermedades cardiacas y cáncer combinadas ${ }^{16}$. Las fracturas del esqueleto facial son un componente común de los politraumatismos como accidentes automovilísticos, accidentes laborales, así como accidentes deportivos a accidentes en actividades diarias, esto los convierte en uno de las fracturas más comunes, además es una condición complicada debido al compromiso de la vía aérea y las posibles hemorragias ${ }^{17,18}$. Los pacientes que acuden con fracturas faciales múltiples, presentan usualmente un peor estado de salud en comparación a los pacientes con lesiones faciales menos severas y presentan un peor pronóstico. Varios análisis de fracturas faciales han sido realizados y los resultados de estos varían de acuerdo al tiempo, región geográfica así como las diferencias sociales, económicas y culturales ${ }^{19}$.

Según Girotto \& col., existe una relación directa entre la gravedad del trauma facial y los reportes de discapacidad laboral ${ }^{20}$. Los pacientes con un traumatismo grave usualmente presentan, en el seguimiento a largo plazo, un mayor porcentaje de discapacidad relacionada con la lesión, como problemas visuales, alteraciones en el olfato, dificultad con la masticación y respiración, entre otras ${ }^{21}$.

El manejo de las fracturas se realizó inicialmente con la utilización de fijación intermaxilar y posterior a esto las fracturas se trataron con fijación interna, es importante en el manejo de las fracturas recrear las condiciones previas del paciente, tanto estructuralmente, la forma, el perfil y la función ${ }^{18}$.

Este estudio evalúa la incidencia de fracturas maxilofaciales basado en 432 pacientes, durante un periodo de 5 años registrado en el hospital Carlos Andrade Marín, los cuales han sido tratados de acuerdo a cada lesión. En nuestro estudio la relación entre hombres $(89,4 \%)$ y mujeres $(10,6 \%)$ fue de $9: 1$, los que concuerda con algunos estudios como el de Gassner \& col. $^{16}$, en el cual la proporción fue de 2:1, el estudio de Kaura \& col. ${ }^{1}$, en el cual fue de $4: 1^{19}$, Gaddipati \& col. ${ }^{15}$, presenta un proporción de 5,5:1 y Abosadegh y Rahman ${ }^{22}$ con 2:1, esto demuestra una mayor frecuencia global en las fracturas maxilofaciales observadas en hombres en comparación con mujeres, esto puede explicarse a que los hombres son más propensos a situaciones de riesgo como accidentes automovilísticos por la mayor cantidad de conductores masculinos en las carreteras, los hombres practican una mayor cantidad de deportes de contacto, el consumo de alcohol que a menudo resulta en peleas y violencia interpersonal.

La edad media de las fracturas faciales en este análisis fue de 34,09 años, lo cual concuerda con otros estudios como el de Gaddipati \& col..$^{15}$., en el cual la edad promedio fue de 31,19 años o el de abosadegh y rahman ${ }^{22}$ que presento un promedio de edad de 29,5 años, aunque los valores reportados en este estudio son relativamente altos comparados con otros estudios como el de Gassner \& col. ${ }^{16}$, en el cual la edad media era de 25.8 años, esta mayor proporción de traumatismo faciales en 
la segunda y tercera década de la vida quizá se deba a que la actividad es mayor lo que los predispone a un mayor riesgo de sufrir accidentes ${ }^{1,22,23}$.

Existen varios estudios que concluyen la causa más común de las fracturas del macizo facial son los accidentes de tránsito ya sea automovilísticos o en motocicleta, y dentro de estos los accidentes en motocicletas sin una adecuada protección son los que presentan un mayor porcentaje ${ }^{15,21}$. Entre los varones $(89,4 \%)$ los accidentes en motocicletas (22,54\%) ocupan el motivo más frecuente de fracturas en accidentes de tránsito, por otro lado, los accidentes en carro $(11,66 \%)$ son los menos frecuentes.

De acuerdo a lo establecido por la Organización Mundial de la Salud, los accidentes de tránsito son la causa más común de traumatismo faciales, esto puede atribuirse a la disponibilidad de automóviles a jóvenes, al exceso de velocidad, las malas condiciones de las carreteras, no portar cascos o equipamiento de seguridad, el no acatar las normas de tránsito y a la influencia del alcohol, sin embargo el porcentaje de accidentes automovilísticos ha decrecido considerablemente debido a la implementación de nuevas leyes de tránsito, aumento en la extensión de las vías, la cultura por parte de la sociedad para incentivar el uso del cinturón de seguridad y un mayor control por parte de las autoridades ${ }^{15,1}$. Esto fue demostrado por Van Beek \& col. ${ }^{24}$ en su estudio longitudinal, en el cual en un periodo de 20 años las fracturas faciales relacionadas con accidentes de tránsito disminuyeron mientras que las fracturas relacionadas con la violencia aumentaron, así mismo Fasola \& col. ${ }^{25}$ mostro en un estudio comparativo en los periodos de 1978-1982 y 1995-1999, en estos periodos los accidentes de tránsito decrecieron del $77,4 \%$ al $69,2 \%$, mientras que las fracturas por agresiones físicas aumentaron del $8,8 \%$ al $12 \%{ }^{26,21}$. Esta disminución en cuanto a las causas se ve reflejado en nuestro estudio en el cual los accidentes de tránsito se presentaron en un $35,4 \%$, mientras que las agresiones físicas fueron en aumentó como causa con un $28,5 \%$.

El factor que menos fracturas faciales causa son los accidentes en bicicleta y los arrollamiento ambos con un porcentaje de $3 \%$, por otro lado se observa un aumento en los accidentes producidos actividad deportiva (9,3\%), esto contrasta por lo expuesto con Gadipatti \& col. ${ }^{15}$ y al contrario concuerda con Gassner \& col. ${ }^{16}$, este aumento en las estadísticas quizá se deba al aumento en la actividad deportiva de las personas para mantener un estilo de vida más saludable.

Entre varios estudios realizados se observa una relación en cuanto al consumo de alcohol como un factor determinante en las agresiones física, accidentes de tránsito y caídas, en nuestro estudio los pacientes que se encontraban en estado de embriaguez fueron del $9,5 \%$, de estos el $26,83 \%$ sufrieron accidentes de tránsito, el $51,22 \%$ sufrieron agresiones físicas y el $17,7 \%$ sufrieron caídas $19,25,27,10$.

En este estudio se observó un mayor número de fracturas del tercio medio (64,6\%) en relación a las fracturas mandibulares $(32,4 \%)$ y del tercio superior facial $(3 \%)$ en una proporción de 1,8:1, resultados similares fueron presentados por Dube \& col. ${ }^{28}$ y Gandhi \& col..$^{29}$ en los cuales la mayoría de los traumatismos se concentraron en el tercio medio y superior facial, por otro lado estudios realizados por Bergh \& col. 
en Holanda ${ }^{17}$ y Kaura \& col. en la India', indicaron que las fracturas mandibulares presentaban una mayor prevalencia que las fracturas de tercio medio.

De las fracturas mandibulares el área más afectada fue el cóndilo, seguido de las fracturas parasinfisiarias esto puede ser debido a que durante los accidentes la mayoría de los pacientes con facturas mandibulares experimentan caídas directas sobre el mentón, lo que produce fracturas combinadas de la sínfisis, parasínfisis y el cóndilo, esto se debe a que el contacto directo en la zona anterior de la mandíbula produce dispersión de las fuerzas hacia el cóndilo, lo que resulta en fracturas indirectas, además la parasínfisis que es una área anatómica de debilidad por la longitud de la raíz del canino ${ }^{15}$. Este tipo de fractura coincide, con otros estudios como lo expuesto por Kaura \& col. ${ }^{1}$, Bergh \& col. ${ }^{77}$ y por Gaddipati \& col..$^{15}$ en los cuales las fracturas de cóndilo son las más comunes, esto puede deberse a el mecanismo del trauma y a la distribución de la fuerza durante el traumatismo. En un estudio realizado por Lee \& col. ${ }^{30}$ se demuestra que la causa más frecuente de las fracturas mandibulares resulto ser la agresión física, en especial de las fracturas del ángulo mandibular, lo que va en desacuerdo con nuestro estudio en el cual la causa más común fueron los accidentes en motocicleta, en la región del cóndilo mandibular.

Por otro lado en el tercio medio y superior, el patrón más común de fracturas fue el del complejo orbito cigomático maxilar $(28,5 \%)$, seguido de fracturas del piso de orbita $(14,10 \%)$ aisladas y arco cigomático $(9,70 \%)$, resultados similares a los presentados por Kirgidis \& col. ${ }^{31}$.

Lo niños representan un grupo especial de pacientes, ellos tienen diferencias significativas con los adultos en lo que respecta al esqueleto facial, el menor tamaño de los maxilares, el pequeño volumen de los senos paranasales, el potencial de crecimiento, la presencia de gérmenes dentarios durante la dentición primaria y mixta, un proceso de cicatrización más rápido y la dificultad de cooperación ${ }^{31,21}$. En nuestro estudio el 7,64\% de los pacientes fueron niños, este resultado es similar a lo presentado por Ghosh \& col. ${ }^{21}$, en el cual el reporte fue de $75 \%$, con una mayor proporción de niños en comparación a niñas de 3:1. Como reportan estudios previos la causa más frecuente fueron las caídas de propia altura $(35,29 \%)$ seguido de actividades deportivas y agresiones físicas $(14,71 \%)$, además existió un predominio de las fracturas mandibulares, seguido de las fracturas del complejo orbito cigomático maxilar ${ }^{18}$.

Varios factores influyen en estos resultados como son exposición a ambientes peligrosos, edad de los padres, educación de los padres, problemas mentales e inequidades a accesos de sistemas de salud, en casos en los cuales los niños son parte de la fuerza laboral, es uno de los riesgos más altos de caídas, debido a que la demanda en el trabajo exige más de lo que su fuerza, capacidades y edad lo permite ${ }^{18}$.

Las fracturas del tercio medio facial $(35,12 \%)$ se presentaron en mayor proporción cuando los pacientes sufrieron accidentes de tránsito, la causa puede deberse a la ausencia de bolsas de aire en los vehículos, lo que deja que se produzcan impactos directos en el tercio medio facial. En cuanto a las fracturas ocasionadas por agresiones físicas, las fracturas del tercio medio (31,18\%) fueron las más comunes seguidas por las fracturas del tercio inferior $(22,14 \%)$, se puede observar esta pro- 
porción debido a que para producir fracturas del tercio medio se requiere de una menor fuerza que en el tercio inferior.

Las fracturas panfaciales se produjeron en un porcentaje de 0,5\% y las fracturas del tercio superior en un porcentaje del $3 \%$.

Como se conoce que la cara presenta butress horizontales y verticales, en donde el esqueleto facial es más grueso y su función es distribuir las fuerzas aplicadas, por lo que la reducción y fijación adecuada de los butress son clave en la reconstrucción del macizo facial'.

\section{Conclusiones}

- Los pacientes de grupos etarios entre los 20 años y 40 años fueron los más propensos a sufrir traumas faciales.

- Dentro de las lesiones más prevalentes, las fracturas mandibulares fueron las que más se presentaron

- La causa más común de traumatismos faciales fue la agresión física.

- El género más afectado es el masculino, esto puede explicarse a que los hombres son más propensos a situaciones de riesgo como accidentes automovilísticos, a que practican una mayor cantidad de deportes de contacto, el consumo de alcohol que a menudo resulta en peleas y violencia interpersonal 


\section{Referencias bibliográficas}

1. Kaura, S., Kaur, P., Bahl, R., Bansal, S., Sangha, P. Retrospective Study of Facial Fractures. Annal of maxillofacial surgery. 2018; 8(1): 78-82.

2. Gassner, R., Bosch, R., Emshoff, R. Prevalence of dental trauma in 6000 patients with facial injuries: implications for prevention. Oral Surg Oral Med Oral Pathol Oral Radiol Endod. 1999; 87(7): 27-33.

3. Yang, C., Chen, S., Yang, Y., Huang, L., Guo, H., Yang, H. Epidemiology and patterns of facial fractures due to road traffic accidents in Taiwan: A 15-year retrospective study. Traffic injury prevention. 2017; 18(7): 724-729.

4. Bonavolonta, P., Dell'aversana, G., Abbate, V., Vaira, L., Lo Faro, C., Pretrocelli, M., Attanasi, F., De Rui, G., Laconetta, G., Califano, L. The epidemiological analysis of maxiIlofacial fractures in Italy: The experience of a single tertiary center with 1720 patients. Journal of craniomaxillofacial surgery. 2017; 45(8): 1319-1326.

5. Almeida, B., Sanches, R., Lanza, E., Andrade, I., Moreira, S., Rocha, C. Prevalence of Maxillofacial Traumas in a Hospital of the Interior of Brazil. Journal of dental health, oral disorders \& therapy. 2017; 7(6): 1-4.

6. Lee, K., Chua, J. Psychosocial Support Following Maxillofacial Trauma and its Impact on Trauma Recurrence. Journal of maxillofacial oral surgery. 2016; 17(1): 32-37.

7. Qudeimat, M., Alhasan, A., AlHasan, M., Al-Khayat, K., Andersson, L. Prevalence and severity of traumatic dental injuries among young amateur soccer players a screening investigation. Dental traumatology. 2019; 0(1).

8. Tent, P., Juncar, R., Lung, T., Juncar, M. Midfacial Fractures: A Retrospective Etiological Study over a 10 Year Period in Western Romanian Population. NIgerian Journal of Clinical Practice. 2018; 21(12): 1570-1575.

9. Rothweiler, R., Bayer, J., Zwingmann, J., Suedkamp, N., Kalbhenn, J., Schmekzeisen, R., Gutwald, R. Outcome and complications after treatment of facial fractures at different times in polytrauma patients. Journal of Cranio-Maxillo-Facial Surgery. 2017; 46(2): 283287.

10. Lee, K., Qiu, M., Sun, J. Temporal distribution of alcohol related facial fractures. Oral surgery, oral medicine, oral pathology, oral radiology. 2017; 124(5): 450-455.

11. Hausamen, J. The scientific development of maxillofacial surgery in the 20th century and an outlook into the future. Journal of craniomaxillofacial surgery. 2001; 29(1): 2-21.

12. Cleveland, P., Ellis, J. Complications of Facial Trauma of the Fronto-orbital Region. Facial plastic surgery. 2017; 33(6): 581-590.

13. Chukwulebe, S., Hogrefe, C. The Diagnosis and Management of Facial Bone Fractures. Emergency Medicine Clinics of North America. 2019; 37(1): 137-151.

14. Pita, I., Pereira, J., Araujo, J., Ramos, M., Abreu, L., Pinheiro, I., Studart, E., Alencar, D., Reis, L. Factors Associated With the Complexity of Facial Trauma. The journal of craniofacial surgery. 2018; 29(6): e562-e566.

15. Gaddipati, R., Ramisetti, S., Vura,N., Reddu, K., Nalamolu, B. Analysis of 1,545 Fractures of Facial Region-A Retrospective Study. Craniomaxillofacial trauma recosntructive. 2015; 8(4): 307-314.

16. Gassner, R., Tuli, T., Hachl, O., Rudisch, A., Ulmer, H. Cranio-maxillofacial trauma: a 10 year review of 9543 cases with 21067 injuries. Journal of Cranio-Maxillofacial Surgery. 2003; 31(1): 51-61. 
17. Bergh, B., Karagozoglu, H., Haymans, M., Forouzanfar, T. Aetiology and incidence of maxillofacial trauma in Amsterdam: A retrospective analysis of 579 patients. Journal of Cranio-Maxillo-Facial Surgery. 2012; 40(1): e165-e169.

18. Haq, M., Khan, A. A retrospective study of causes, management, and complications of pediatric facial fractures. European Journal of Dentistry. 2018; 12(2): 247-252.

19. Hoon, J., Cho, B., Park, W. A 4-year retrospective study of facial fractures on Jeju, Korea. Journal of Cranio-Maxillo-Facial Surgery. 2010; 38(1): 192-196.

20. Girotto, J., Mackenzie, E:, Fowler, C., Redett, R., Robertson, B., Manson, P. Long-term physical impairment and functional outcomes after complex facial fractures. Plastic and reconstructive surgery. 2001; 108(2): 312-327.

21. Ghosh, R., Gopalkrishnan, K. Facial Fractures. Journal of craniofacial surgery. 2018; 29(4): 334-340.

22. Abosadegh, M., Rahman, S. Epidemiology and Incidence of Traumatic Head Injury Associated with Maxillofacial Fractures: A Global Perspective. Journal of international oral health. 2018; 10(2): 63-70.

23. Mosaddad, S., Gheisari, R., Erfani, M. Oral and maxillofacial trauma in motorcyclists in an iranian subpopulation. Dental Traumatology. 2018; 34(5): 347-352.

24. Van Beek, G., Merkx, C. Changes in the pattern of fractures of the maxillofacial. International journal of oral and maxillofacial surgery. 1999; 6(1): 424-428.

25. Fasola, A., Nyako, E., Obiechina, A., Arotiba, J. Trends in the characteristics of maxillofacial fractures in Nigeria. Journal of oral and maxillofacial surgery. 2003; 61(10): 11401143.

26. Subhashraj, K., Nandakumar, N., Ravindran, C. Review of maxillofacial injuries in Chennai, India: a study of 2748 cases. British journal of oral and maxillofacial surgery. 2007; 45(8): 637-639.

27. Hutchison, I., Magennis, P., Shepherd, J., Brown, A. The BAOMS United Kingdom survey of facial injuries part 1: aetiology and the association with alcohol consumption. British Association of Oral and Maxillofacial Surgeons. British journal of oral and maxillofacial surgery. 1998; 36(1): 3-13.

28. Dube, A., Rao, G., Tanwar, A. Pattern of Maxillofacial Injury Associated With Head Injury at a Neuro Surgical Centre: An Analysis of 250 Cases. International journal of dental and medical speciality. 2014; 1(2): 2-6.

29. Gandhi, S., Ranganathan, L., SOlanki, M., Mathew, G., Singh, L., Bither, S. Pattern of maxiIlofacial fractures at a tertiary hospital in Northern India: A 4year retrospective study of 718 patients. Dental Traumatology. 2011; 27(4): 257-262.

30. Lee, K. Interpersonal violence and facial fractures. Journal of oral and maxillofacial fractures. 2009; 67(9): 1878-1883.

31. Kyrgidis, A., Koloustsos, G., Kommata, A., Lazarides, N., Antoniades, K. Incidence, aetiology, treatment outcome and complications of maxillofacial fractures. A retrospective study from Northern Greece. Journal of craniomaxillofacial surgery. 2013; 41(7): 637-643. 